\title{
ON A DIOPHANTINE EQUATION
}

\section{FLORIAN LUCA}

In this note, we find all solutions of the diophantine equation $x^{2}+3^{m}=y^{n}$, where $(x, y, m, n)$ are non-negative integers with $x \neq 0$ and $n \geqslant 3$.

In this note, we investigate the equation

$$
x^{2}+3^{m}=y^{n}
$$

when $x>0$ and $n \geqslant 3$.

For $n=2$ the problem is not interesting because in this case the given equation (1) has infinitely many solutions and all of them are of the form

$$
\left\{\begin{array}{l}
x=\frac{3^{a}-3^{b}}{2}, \\
y=\frac{3^{a}+3^{b}}{2}, \\
m=a+b,
\end{array} \text { for some integers } a>b \geqslant 0 .\right.
$$

The fact that equation (1) has no solution when $m=0$ was shown by Lebesgue (see [7]) and the fact that (1) has no solution for $m=1$ was proved by Cohn (see [4]). Recently, Arif and Muriefah (see [1]) found all solutions of equation (1) when $m$ is odd. They are all of the form $x=10 \cdot 3^{3 t}, y=7 \cdot 3^{2 t}, m=5+6 t$ and $n=3$. The same authors investigated equation (1) for $m$ even in [2].

Our result is the following:

THEOREM. All solutions of equation (1) with $m$ even are of the form $x=46 \cdot 3^{3 t}$, $m=4+6 t, y=13 \cdot 3^{2 t}$ and $n=3$.

We begin by showing that it suffices to treat equation (1) when $3 \nmid x$. Indeed, assume that $x=3^{a} x_{1}$ for some $a \geqslant 1$ and $3 \nmid x_{1}$. Write $y=3^{b} y_{1}$ where $b \geqslant 0$ and $3 \nmid y_{1}$. Equation (1) becomes

$$
3^{2 a} x_{1}^{2}+3^{m}=3^{n b} y_{1}^{n}
$$

\section{Received 26th May, 1999}

Financial support from the Alexander von Humboldt Foundation is gratefully acknowledged. We would like to thank Yuri Bilu for sending us a copy of the preprint [3]. We would also like to thank Professor Andreas Dress and his research group in Bielefeld for their hospitality during the period when this paper was written.

Copyright Clearance Centre, Inc. Serial-fee code: 0004-9727/00 \$A2.00+0.00. 
We distinguish 3 cases:

CASE 1. $2 a>m$.

Equation (2) becomes

$$
\left(3^{a-m / 2} x_{1}\right)^{2}+1=3^{n b-m} y_{1}^{n}
$$

From equation (3) it follows that $n b=m$. If we denote by $X=3^{a-m / 2} x_{1}$ and by $Y=y_{1}$, we get

$$
X^{2}+1=Y^{n}
$$

which has no solution by Lebesgue's result.

Case 2. $2 a=m$.

Equation (2) becomes

$$
x_{1}^{2}+1=3^{n b-m} y_{1}^{n}
$$

Since -1 is not a quadratic residue modulo 3 , it follows that $n b=m$. Hence, equation (5) becomes

$$
x_{1}^{2}+1=y_{1}^{n}
$$

which is again Lebesgue's equation.

Case 3. $2 a<m$.

Equation (2) becomes

$$
x_{1}^{2}+3^{m-2 a}=3^{n b-2 a} y_{1}^{n}
$$

From equation (6), it follows that $n b=2 a$. Equation (6) is now

$$
x_{1}^{2}+3^{m_{1}}=y_{1}^{n}
$$

with $m_{1}=m-2 a$ even. Equation (7) is precisely equation (1) for $m_{1}$ even and $3 \nmid x_{1}$.

From now on we assume that $(x, y, m, n)$ is a solution of (1) with $3 \nmid x$. Notice that $x$ is even and that $y$ is odd - indeed, if $x$ is odd then $x^{2}+3^{m} \equiv 2(\bmod 8)$, hence it cannot be the power of an even number (nor of a odd number). 
We treat two cases:

\section{The CASE $4 \mid n$}

In this case, we may assume that $n=4$. Equation (1) can be rewritten as

$$
3^{m}=\left(y^{2}-x\right)\left(y^{2}+x\right)
$$

Since $y^{2}-x$ and $y^{2}+x$ are coprime, it follows that

$$
\begin{aligned}
& y^{2}-x=1 \\
& y^{2}+x=3^{m}
\end{aligned}
$$

Hence, $2 y^{2}=3^{m}+1$ or

$$
\left(3^{m / 2}\right)^{2}-2 y^{2}=-1
$$

The equation

$$
X^{2}-2 Y^{2}=-1
$$

is a Pell equation and its positive solutions are given by $X_{1}=1, Y_{1}=1, X_{2}=7$, $Y_{2}=5$ and

$$
X_{n}=6 X_{n-1}-X_{n-2}, Y_{n}=6 Y_{n-1}-Y_{n-2}
$$

It follows that $3 \nmid X_{n}$, which contradicts the fact that $X=3^{m / 2}$.

Thus, equation (1) has no solution such that $4 \mid n$.

\section{The CASE $4 \nmid n$}

Since $n \geqslant 3$ and $4 \nmid n$, it follows that there exists an odd prime $p$ such that $p \mid n$. We may assume that $n=p$. Equation (1) becomes

$$
x^{2}+3^{m}=y^{p}
$$

Since $x^{2} \equiv y^{2} \equiv 1(\bmod 3)$, it follows that $y \equiv 1(\bmod 3)$. Rewrite equation $(11)$ as

$$
\left(x+i 3^{m / 2}\right)\left(x-i 3^{m / 2}\right)=y^{p} .
$$

Since $Z[i]$ has class number 1 and $\operatorname{gcd}\left(x+i 3^{m / 2}, x-i 3^{m / 2}\right)=1$, it follows that the exists two integers $a$ and $b$ such that $y=a^{2}+b^{2}$ and

$$
\left\{\begin{array}{l}
x+i 3^{m / 2}=(a+i b)^{p} \\
x-i 3^{m / 2}=(a-i b)^{p} .
\end{array}\right.
$$


Notice that $a b \neq 0$. Solving system (12), we get

$$
\begin{aligned}
x & =\frac{(a+i b)^{p}+(a-i b)^{p}}{2}, \\
3^{m / 2} & =\frac{(a+i b)^{p}-(a-i b)^{p}}{2 i} .
\end{aligned}
$$

Since $p$ is odd, it follows from the first equation (13) that $a \mid x$. In particular, $3 \nmid a$. Moreover, from the second equation (13), it follows that $b \mid 3^{m / 2}$.

We treat first the case $p=3$. In this case, the second equation (13) becomes

$$
3^{m / 2}=b\left(3 a^{2}-b^{2}\right)
$$

Reducing equation (14) modulo 3 , it follows that $3 \mid b$. In particular, $9 \mid b\left(3 a^{2}-b^{2}\right)$ which gives $m / 2 \geqslant 2$. If $m / 2=2$, we get

$$
9=b\left(3 a^{2}-b^{2}\right)
$$

and $b= \pm 3$. This leads to $a=2, b=3$, which gives the solution $(x, y, m, n)=$ $(46,13,4,3)$ which was previously found by Cohn (see [5]).

We now show that equation (14) has no solution for $m>4$. Indeed, let $b= \pm 3^{u}$ for some $u, 0<u<m / 2$. Equation (14) becomes

$$
3 a^{2}-3^{2 u}= \pm 3^{m / 2-u}
$$

or

$$
a^{2}=3^{2 u-1} \pm 3^{m / 2-u-1} \text {. }
$$

Since $3 \nmid a$, it follows that $u=m / 2-1$ and

$$
a^{2}=3^{m-3} \pm 1
$$

The equation with -1 leads to

$$
a^{2}+1=3^{m-3}
$$

for some $m \geqslant 6$ which is impossible by Lebesgue's result. The equation with +1 leads to

$$
a^{2}=3^{m-3}+1
$$

with $m \geqslant 6$. From a result of Chao Ko (see [6]), we know that the only nontrivial solution of the equation

$$
X^{2}=Y^{n}+1
$$


for some $n \geqslant 3$ is given by $X=3, Y=2$. Hence, equation (17) has no solution.

From now on we assume that $p>3$. We first show that $b= \pm 3^{m / 2}$. Notice first that $b \neq \pm 1$. Indeed, if $b= \pm 1$, then $y=a^{2}+b^{2}=a^{2}+1$. Since $y \equiv 1(\bmod 3)$, it follows that $a \equiv 0(\bmod 3)$ which is a contradiction. Hence, $b= \pm 3^{u}$ for some $u, 0<u \leqslant m / 2$. Assume that $b= \pm 3^{u}$ for some $u<m / 2$. After simplifying the second equation (13) by $b$ and reducing it modulo 3 we get $p a^{p-1} \equiv 0(\bmod 3)$ which is impossible for $p>3$ prime and $3 \nmid a$. Hence, $b= \pm 3^{m / 2}$. From [5, Lemma 4 and Lemma 5], it follows that $b=-3^{m / 2}, C=3^{m} \equiv 1(\bmod 16)$ and $p \equiv-1(\bmod 12)$. In particular, $4 \mid \mathrm{m}$. From the same paper of Cohn, we also know that $a$ is even and that if $q$ is any odd prime dividing $a$, then

$$
3^{m(q-1)} \equiv 1\left(\bmod q^{2}\right)
$$

and that if $q^{\alpha} \| a$, then $q^{2 \alpha} \|\left(3^{m(q-1)}-1\right)$.

We now return to the second equation (13). Let $\varepsilon=a+i b$ and $\bar{\varepsilon}=a-i b$. Since $b=-3^{m / 2}$, it follows that

$$
\frac{\varepsilon^{p}-\bar{\varepsilon}^{p}}{\varepsilon-\bar{\varepsilon}}=-1
$$

Notice that the sequence

$$
u_{k}=\frac{\varepsilon^{k}-\bar{\varepsilon}^{k}}{\varepsilon-\bar{\varepsilon}} \quad \text { for all } k \geqslant 0
$$

is a Lucas sequence. By the results of [3], we know that in this case $u_{k}$ has a primitive divisor for all prime values of $k>13$. Moreover, for $k \in\{5,7,11,13\}$ there are precisely 10 Lucas sequences for which $u_{k}$ does not have a primitive divisor and all these 10 sequences can be found in [3, Table 1]. One can easily see that none of these 10 sequences has the property that the roots of the characteristic equation are in $\mathbf{Z}[i]$. Hence, $\left|u_{p}\right|>1$, which contradicts (19). It follows that there are no solutions for $p>3$.

One can now employ the arguments from Case 3 at the beginning of the paper to conclude that the general solution of equation (1) for $m$ even is given by $x=46 \cdot 3^{3 t}$, $m=4+6 t, y=13 \cdot 3^{2 t}$ and $n=3$.

\section{REFERENCES}

[1] S.A. Arif and F.S.A. Muriefah, 'The diophantine equation $x^{2}+3^{m}=y^{n}$, Internat. $J$. Math. Math. Sci. 21 (1998), 619-620.

[2] S.A. Arief and F.S.A. Muriefah, 'On a diophantine equation', Bull. Austal. Math. Soc. 57 (1998), 189-198. 
[3] Y. Bilu, G. Hanrot and P.M. Voutier, 'Existence of primitive divisors of Lucas and Lehmer numbers', (preprint).

[4] J.H.E. Cohn, 'The diophantine equation $x^{2}+3=y^{n}$, Glasgow Math. J. 35 (1993), 203-206.

[5] J.H.E. Cohn, 'The diophantine equation $x^{2}+c=y^{n}$ ', Acta. Arith. 65 (1993), 367-381.

[6] Chao Ko, 'On the diophantine equation $x^{2}=y^{n}+1, x y \neq 0$ ', Sci. Sinica 14 (1965), 457-460.

[7] V.A. Lebesgue, 'Sur l'impossibilité en nombres entiers de l'equation $x^{m}=y^{2}+1$ ', Nouv. Annal. des Math. 9 (1850), 178-181.

Mathematical Institute

Czech Academy of Sciences

Žitná 25, 11567 Praha 1

Czech Republic

e-mail: luca@math.cas.cz 